\title{
A step towards resolving the mystery of HPV vaccination: breakthrough of cervical cancer
}

\begin{abstract}
Cervical cancer is the second most prevalent cancer in women worldwide, with about 500,000 cases and over 270,000 deaths estimated annually. The disease is caused due to the persistent infection of one or more type of high risk HPVs in which HPV16/18 are the most common. In India, HPV-16 is the most prevalent type of High risk HPV associated with cervical cancer risk. In the year 2006-2007, two VLP based prophylactic HPV vaccines, Gardasil and Cervarix came into the market. Recently in 2014, a new nonavalent vaccine Gardasi19 has been licensed as the improvised form of tetravalent Gardasil by FDA. Though HPV vaccines prevent its spread and persistence but they are insufficient in reverting or eliminating the already established invasive cancers. Besides, these vaccines are also having certain limitations which includes require refrigeration, higher cost, limited serotype and efficacy is person dependent. To overcome the limitations of the available vaccines, the development of second generation vaccines e.g. capsomere based vaccines, plant based edible vaccines, recombinant live-vector vaccines, protein and peptide based vaccines, multi-epitopic based vaccines and DNA-based vaccines is under way. These vaccines will be heat stable, cost effective and may apply both in prevention and also in the treatment of cancers. It has been stated that cervical cancer is detected mainly in women older than 35 years suggesting HPV infection at a younger age and slow progression to cancer at an older age. It is really difficult to promote vaccination for younger girls in countries like India to prevent the onset of the disease because of the cultural and prestigious issues related to STDs. So there is an urging need in India for the development of therapeutic vaccine for the eradication of the devastating disease as well as to meet the global responsibility of achieving herd immunity.
\end{abstract}

Keywords: HPV, cervical cancer, vaccine, VLP, DNA vaccine
Volume 3 Issue I - 2018

\author{
Mausumi Bharadwaj,' Heena Gautam,' Pallavi \\ Singhal,' Anoop Kumar ${ }^{2}$ \\ 'Department of Molecular Genetics and Biochemistry, National \\ institute of Cancer Prevention and Research, India \\ ${ }^{2}$ National institute of Biological, India
}

\begin{abstract}
Correspondence: Mausumi Bharadwaj, Scientist F (Sr Deputy Director) \& Co-ordinator, Molecular Biology Group, National Institute of Cancer, Prevention \& Research (Formerly, Institute of Cytology \& Preventive Oncology), Indian Council of Medical Research (ICMR), Department of Health Research (Ministry of Health \& Family Welfare, Govt of India), I-7, Sector 39, Noida, India, Fax 0I 20 - 2579473, Tel 0I20-24469I0 (O), Email mausumi.bharadwaj@gov.in, mausumi.bharadwaj@gmail.com
\end{abstract}

Received: November 20, 2017 | Published: January 12, 2018

\section{Introduction}

HPVs (Human Papilloma virus) are associated with a variety of clinical conditions that ranges from less harmful lesions to life threatening cancer. During the onset of $19^{\text {th }}$ century, HPV association with human cancers has been found with major links to genital warts and cervical cancers. ${ }^{1}$ With recent advances in HPV vaccine, the possibility of preventing the second most common cancer among the women is promising. But as these vaccines are prophylactic vaccines there is a dynamic need for the development of therapeutic vaccine. In this review, we would discuss about HPV and Cervical cancer, immunological aspects of HPV infection, current vaccines and vaccine case studies, recent strategies employed for the development of therapeutic vaccines with emphasis on second generation vaccine and its future prospects.

\section{Discussion}

\section{HPV and cervical cancer}

Every year, there are122,844 new cases of cervical cancer in India as compared to 527624 cases worldwide that almost contribute to onefourth of cervical cancer burden globally. ${ }^{2}$ Cervical cancer is often the most common cancer in women of developing countries constituting up to $25 \%$ of all female cancers. Papillomaviruses (PVs) are present almost everywhere and are hosted by many animals as well as humans to which they are specific for and is considered the most common cause of cervical cancer. ${ }^{3}$ There are many other risk factors that can cause cervical cancer like the early age of sexual activity, multiple sexual partners, multiple pregnancies, poor genital hygiene, cigarette smoking, oral contraceptives, HIV and immune suppressive drugs etc. ${ }^{4}$ HPVs exhibit a great diversity in their genomic sequences because of which there are more than two hundred variants. ${ }^{3}$ Based on their association with cervical cancer and precursor lesions, HPVs have been grouped into high-risk and low-risk HPV types. Types 6 , $11,42,43$, and 44 comes under low-risk HPVs. High-risk HPV types include types $16,18,31,33,34,35,39,45,51,52,56,58,59,66,68$, and $70 .{ }^{5}$ Both the HR-HPVs (HPV-16 and HPV-18) being the most prominent HPVs contributing to over in $>95 \%$ of cervical cancer cases ${ }^{6}$ and HPV16 alone accounts for more than half the cases of cervical cancer worldwide. High-risk HPV establishes its genome as an extrachromosomal episome by infecting the basal cells in the cervical epithelium through microlesions. ${ }^{7}$ Some molecular studies have revealed fundamental mechanisms of HPV carcinogenesis by which HPV replicates, transforms cells and evades the immune system. HPV is a nonenveloped small virus of $55 \mathrm{~nm}$ diameter. It is a polyhedron capsid composed of 72 capsomers, the genome contains three regions: a noncoding upstream regulatory region, the long control region (LCR), an early region, consisting of ORFs E1, E2, E4, E5 (involved in viral replication), E6 and E7 (involved in oncogenesis) and a late region, which encodes the structural proteins for viral capsid L1 and L2 ${ }^{8}$ The HPV E7 protein is an $11 \mathrm{kDa}$ protein that binds with the $\mathrm{pRb}, \mathrm{p} 107$ and $\mathrm{p} 130$ proteins leading to dissociation of the E2F family of transcription factors, which are required for the transactivation of cellular proteins cyclins $\mathrm{A}$ and $\mathrm{E}$ involved in replication. 
Another is E6 protein $(16 \mathrm{kDa})$ which associates with p53 and leads to its ubiquitination and degradation further escape growth arrest and anti-apoptotic role by associating with Bak protein also. ${ }^{9}$ Other than HPV there are other viruses such as human herpesvirus serve as co-factors in the development of cervical cancer. Besides this there are numerous other factors involved in cervical cancer progression such as heritability including vulnerability to HPV infection, ability to abolish HPV infection, time taken for developing the disease and infections with multiple types of HPV. ${ }^{1}$ It is observed that the infection with one type of HPV variant in a tissue changed its micro environment in such a way that it became much sensitive for the co- infection with the multiple HPV genotypes. As reported the rate of multiple-infection seen up to $39 \%$ could lead to the severity of cervical disease as it comprises of $34 \%$ infection with high risk HPVs. ${ }^{10}$ Another approach that is also recognized recently is the concept of quasispecies. The intratypic variants of same genotypes are considered quasispecies which have originated either by co-infection or by mutegenesis arising from single variant. ${ }^{7,11}$ Have reported multiple variants of Indian specific HPV 16 (V1-V16). Although these variants carry minor genetic variations in their genome but in future these key changes could provide novel strategies for the diagnosis and clinical management of HPV 16 infections. Pathogenicity of HPV variants differs according to their properties. It is also observed that the oncogenicity of specific HPV variants appears to vary geographically and also with the ethnic origin of the population studied. ${ }^{12}$

\section{Immunological aspects of HPV infection}

In most of the circumstances, final resolution of a pathogenic infection necessitates the initiation of a local inflammatory response; suitably assent to provide the recruitment of adaptive immune effectors to deal with the danger. These early steps are part of the nonspecific innate immune response which is responsible for local damage and specific pathogen associated molecular patterns engage with pattern recognition receptors on APCs for activation of the local antigen milieu. Afterward, these APCs migrate to local secondary immune sites where these can activate relevant antigen-specific $\mathrm{T}$ cells, which then can be recruited to the inflammatory site. ${ }^{13}$ A sequence of inter-connected procedures combine in neoplastic progression tries to promote immune deviation and ultimately a metastasizing tumor forms. Although HPV has anti-inflammatory properties, high-grade lesions are infiltrated by myelo-monocytic cells.In this process HPV-transformed cells produce IL-6 which acts on tumourassociated myeloid and inflammatory cells in paracrine manner. ${ }^{14}$ As a consequence of this, STAT3 gets activate in monocytesand translates the signal into CCL2 production(an immune-modulating and tumourpromoting factor). In addition, CCL2-motivated $\mathrm{Ca}^{2+}$ signalling leads to MMP-9 up regulation, which helps in cancer progression. ${ }^{15}$ The combination of local immune factors upregulate the expression of checkpoint-inhibitor ligand such as PD-L1 and this acts as a block to antitumour-specific T-cell effectors. ${ }^{16}$ The enhanced knowledge and understanding of complex interactions of HPV-associated neoplasia has provided opportunities for new or improved strategies for therapeutic intervention. In addition, High Risk (HR) HPV influences immune evasion by several complementary strategies during early infection. The viral HPV E5 hampers with trafficking of MHC class I molecules during a physical interaction, ${ }^{17}$ on the other hand $\mathrm{E} 7$ reduces the density of surface MHC molecules and antigen presentation through its effect on STAT1 signaling and the suppression of IRF$1 .{ }^{18}$ The E6 protein normally associates with Tyk2, thereby impairing Jak-STAT activation by IFN $\alpha \cdot{ }^{19}$ All these effects together cooperate the MHC class processing and presentation of viral peptides at the infected cell surface and possibly desensitize the target susceptibility to IFN. This reduces the visibility of infected cells to innate and adaptive immune-effectors mechanisms favoring viral persistence.

\section{Vaccine}

Cervarix and Gardasil, bivalent and tetravalent vaccines respectively are the commercially available vaccines against HPVs. Firstly experimental monovalent vaccine compound was made by self-assembling the L1 protein as virus like particle (VLP) which was found to be effective against the structurally analogous HPVs such as HPV 16 VLP showed cross reactivity for HPV 31 and HPV 18 for HPV $45 .{ }^{20}$ The VLP based technique was adopted to make first vaccine, a quadrivalent vaccine against HPV 6/11/16/18 came into the market in 2006 by Merck named as Gardasil and Silgard.After that a bivalent vaccine which is the HPV 16 and $18 \mathrm{~L} 1$ protein products conjugated with an adjuvant aluminum salt marketed as Cervarix since 2007 in Europe and 2009 in US. ${ }^{21}$ Both the vaccine has to be given as 3 dose schedule (qHPV- 0, 2, 6 months and bHPV- 0, 1, 6 months) There is shortage in vaccination and even if some women are vaccinated there is struggle to achieve the application of vaccine either in the second dose or for the last dose. Both vaccines showed up to 10 yrs. protection and are expected to provide protection even beyond this period. Now there is a new nonavalent vaccine Gardasil9 that has also been introduced as the improvised form of tetravalent Gardasil which was licensed on December 14, 2014 by FDA for 9-26 yrs. old women and 9-1 5 yrs. old boys. HPV infections and related diseases are common in boys and men too and women generally get infected with HPV through their male counterparts so male vaccination would serve as a great step towards attaining protection for both the genders. Moreover for countries like India, people are really sensitive for vaccinating women against intended for sexually transmitted diseases before marriage so vaccinating males for protection and achieving herd immunity would be simpler. ${ }^{22}$

\section{Case study}

Some countries have taken the initiative to achieve global herd immunity like UK, Australia, US, Denmark and Scotland to set up a wide HPV vaccination program since 2007 for women aged 12-26 yrs. There was observed a notable abolition of genital warts in women within 3 yrs. which lead to mass immunity. ${ }^{20}$

\section{Drawbacks of current vaccines}

I. Though HPV vaccines prevent its spread and persistence but they are insufficient in reverting or eliminating the already established invasive cancers.

II. All the current vaccines in use contain only the L1 or L2 or both proteins whose expression is lost in transformed cells so these vaccine products are insufficient for therapeutic aspects and only work as prophylactic vaccines. ${ }^{23}$

III. Requires refrigeration.

IV. Their high cost limits their use in low resource countries.

V. Limited serotype.

VI. The humoral immunity induced is primarily type specific, so the level of protection will not support complete protection from other oncogenic HPV types. 
VII. The efficacy of the vaccine is person dependent.

\section{Vaccine designs recently under study}

To overcome the limitations of the available vaccines, researchers are trying to develop second generation vaccines e.g. capsomere based vaccines, plant based edible vaccines, recombinant live-vector vaccines, protein and peptide based vaccines, multi-epitopic based vaccines and DNA-based vaccines or "Genetic vaccines". ${ }^{24}$

\section{Chimeric L2-based}

The L2 minor capsid protein are also been in study as these have highly conserved $\mathrm{N}$ - terminal regions which are incorporated into L1 protein to form an immunogenic compound as it is not type restricted as are the current vaccines. But these chimeric compounds can induce low level neutralizing antibodies for not only the homologous types but also cross neutralize the heterologous HPV types. The neutralizing antibodies produced by L1 VLP are highly immunogenic than the L2 chimeric protein because L2 is not exposed over the HPV surface and also may be because L1 dominates L2 in the chimeric form. ${ }^{25}$ Though HPV vaccines prevent its spread and persistence but they are insufficient in reverting or eliminating the already established invasive cancers. All the current vaccines in use contain only the L1 or L2 or both proteins whose expression is lost in transformed cells so these vaccine products are insufficient for therapeutic aspects and only work as prophylactic vaccines, require refrigeration, and their high cost limits their use in low resource countries like India. ${ }^{23}$

\section{Multi-epitopic based}

Recently for colorectal cancer chimaeric viruslike particles (VLP), a form of non-infectious non-replicative subunit vaccine consisting of rabbit haemorrhagic disease virus (RHDV) VP60 capsid proteins containing recombinantly inserted epitopes from murine topoisomerase II $\alpha$ and surviving have been developed. These vaccines were developed in mono- (T.VP60, S.VP60) and multi-target (TS. VP60) forms, aimingto elucidate the potential benefits from multitarget vaccination, this strategy of combining of epitopes was capable of complete tumor eradication so may also be useful for HPV related tumors. ${ }^{26}$

\section{DNA Vaccine encoding HPVI6 (E6 and E7)}

Study has also been done for vaccine development using oncoproteins E6 and E7 that are constantly expressed by HPVassociated premalignant neoplastic epithelial and cancer cells. Therefore, E6 and E7 become optimal antigen targets for immunotherapy. It has been showed that a vaccine covering HPV 16 E6 and E7asa DNA construct induced long-lasting antibody and CD8+ T-cell (CTL) responses in mice that could combat the progress of HPV16-E7-expressing tumors. The construct was combined with PDL1 (immune check point blockade) and it further enhanced antitumor immunity. ${ }^{27} \mathrm{~A}$ new approach for DNA based vaccine suggests the vector that encodes for a TAA (tumor associated antigen) and T-cell costimulatory molecules which can be used for initial priming and the other is for booster effect. Such innovative approaches have been used in other cancers for ensuring a more viable immune response against the TAAs but not yet been used for cervical cancer vaccine. ${ }^{28}$

\section{Advantages}

A. Heat stable so there is no need of refrigeration.

B. Production is easy on a large scale in timely manner and cost effective.
C. DNA vaccines have a promising application both in the prevention of cancer and also in the treatment of already existing cancers.

D. DNA vaccine can be presented by both MHC-I and MHC-II so they have the potential to induce both humoral and cellular response.

E. The activated CD8+ T-cells also release high levels of inflammatory cytokines, such as interferon-(IFN) and tumor necrosis factor- (TNF), to induce a conducive environment for anti-tumor response.

F. The T-cell effector responses by DNA vaccine elicit Th1 biased immune response which helps in preserving durable immune memory and also there is no risk of infection by this technique.

G. DNA vaccines have a promising application both in the prevention of cancer and also in the treatment of already existing cancers.

\section{Conclusion}

Novel therapeutic endeavors utilizing the recent breakthroughs in understanding the immunological details of the infection are need of the hour due to the drawback of current prophylactic vaccines. E6/ E7 being oncogenes should be considered as an ideal target for DNA vaccine development as both genes are expressed in non-transformed as well transformed cells of infected epithelium. Intervention that are likely to contribute for DNA vaccine development can be further enhanced by combining different immunosuppressive components, conjugating adjuvants and T-cell co-stimulatory molecules with therapeutic HPV DNA vaccine constructs. Some studies states that cervical cancer is detected mainly in women older than 35 years suggesting HPV infection at a younger age and slow progression to cancer at an older age. It is really difficult to promote vaccination for younger girls in countries like India to prevent the onset of the disease because of the cultural and prestigious issues related to STDs. So there is an urging need in India for the development of therapeutic vaccine for the eradication of the devastating disease as well as to meet the global responsibility of achieving herd immunity.

\section{Acknowledgements}

Authors like to acknowledge the financial support from ICMR, Govt. India (file No.5/13/19/2014/NCD III).

\section{Conflict of interest}

The author declares no conflict of interest.

\section{References}

1. Das BC, Hussain S, Nasare V, Prospects and Prejudices of Human Papillomavirus Vaccines in India. Vaccine. 2008;26(22):2669-2679.

2. ICO HPV Information Center. Human Papillomavirus and Related Diseases Report - World. Albania: Springer; 2017.

3. Burd E. Human Papillomavirus and Cervical Cancer. Clin Microbiol Rev. 2003;16(1):1-17.

4. Bharadwaj M, Hussain S, Nasare V, et al. HPV \& HPV vaccination: Issues in developing countries. Indian J Med Res. 2009;130(3):327-333.

5. Ibeanu A. Molecular Pathogenesis of Cervical Cancer. Cancer Biology and Therapy. 2011;11(3):295-306. 
6. Chen X, Jiang J, Shen H, et al. Genetic susceptibility of cervical cancer. Journal of Biomedical Research. 2011; 25(3):155-164.

7. Kukimoto I, Muramatsu M. Genetic variations of human papillomavirus type 16: implications for cervical carcinogenesis. Jpn J Infect Dis. 2015;68(3):169-175.

8. Freitas LB, Chen Z, Muqui EF, et al. Human papillomavirus 16 non-european variants are preferentially associated with high-grade cervical lesions. PLoS One. 2014;9(7):e100746.

9. Sen S, Mandal P, Bhattacharya A, et al. Impact of viral and host DNA Methylations on HPV16-related cervical cancer pathogenesis. Tumor Biology. 2017;39(5):101042831769979.

10. Ralston Howe E, Li Z, Mc Glennen RC, et al. Type-specific prevalence and persistence of human papillomavirus in women in the united states who are referred for typing as a component of cervical cancer screening. Am J Obstet Gynecol. 2009;200(3):245.

11. Kumar A, Hussain S, Sharma G, et al. Identification and validation of immunogenic potential of india specific HPV-16 variant constructs: in-silico \&amp; in-vivo insight to vaccine development. Sci Rep. 2015;5:15751.

12. Cornet I, Gheit T, Iannacone MR, et al. HPV16 genetic variation and the development of cervical cancer worldwide. $\mathrm{Br} J$ Cancer. 2012;108(1):240-244.

13. Comerford I, Harata-Lee Y, Bunting MD, et al. A Myriad of Functions and Complex Regulation of the CCR7/CCL19/CCL21 Chemokine Axis in the Adaptive Immune System. Cytokine Growth Factor Rev. 2013;24(3):269-283.

14. Walch-Rückheim B, Mavrova R, Henning M, et al. Stromal fibroblasts induce CCL20 through IL6/C/EBP $\beta$ to support the recruitment of Th17 cells during cervical cancer progression. Cancer Res. 2015;75(24):5248-5259.

15. Brown GT, Murray GI. Current mechanistic insights into the roles of matrix metalloproteinases in tumour invasion and metastasis. J Pathol. 2015;237(3):273-281.

16. Ott PA, Hodi FS, Robert C. CTLA-4 and PD-1/PD-L1 Blockade: New immunotherapeutic modalities with durable clinical benefit in melanoma patients. Clinical Cancer Research. 2013;19(19):5300-5309.
17. Venuti A, Paolini F, Nasir L, et al. Papillomavirus E5: The smallest oncoprotein with many functions. Molecular Cancer. 2011;10:140.

18. Bottley G, Watherston OG, Hiew YL, et al. High-risk human papillomavirus e7 expression reduces cell-surface mhc class i molecules and increases susceptibility to natural killer cells. Oncogene. 2008;27(12):1794-1799.

19. Li S, Labrecque S, Gauzzi MC, et al. The Human Papilloma Virus (HPV)-18 E6 Oncoprotein Physically Associates with Tyk2 and Impairs Jak-STAT Activation by Interferon-Alpha. Oncogene. 1999;18(42):5727-5737.

20. Pils S, Joura EA. From the monovalent to the nine-valent HPV vaccine. Clin Microbiol Infect. 2015;21(9):827-833.

21. Nicolas Schaad, Mentor Ali, Ber Lucien, et al. HHS Public Access. 2016;34(10):1-15.

22. Hussain S, Bharadwaj M, Nasare V, et al. Human papillomavirus infection among young adolescents in india : impact of vaccination. $J$ Med Virol. 2012;84(2):298-305.

23. Ghorban Hosseini N, Tebianian M, Farhadi A, et al. In silico analysis of L1/L2 sequences of human papillomaviruses: implication for universal vaccine design. Viral Immunology. 2017;30(3):210-223.

24. Geraets DT, Van Doorn LJ, Kleter B, et al. Long-term follow-up of HPV16-positive women: persistence of the same genetic variant and low prevalence of variant co-infections. PLOS ONE. 2013;8(11):e80382.

25. Huber B, Schellenbacher C, Shafti-Keramat S, et al. Chimeric L2-Based virus-like particle( VLP) vaccines targeting cutaneous human papillomaviruses ( HPV ). PLoS One. 2017;12(1):e0169533.

26. Donaldson B, Al-Barwani F, Pelham SJ, et al. Multi-target chimaeric vlp as a therapeutic vaccine in a model of colorectal cancer. $J$ Immunother Cancer. 2017;5(1):69.

27. Chandra J, Dutton JL, Li B, et al. DNA Vaccine encoding HPV16 oncogenes E6 and E7 induces potent cell-mediated and humoral immunity which protects in tumor challenge and drives E7-expressing skin graft rejection. J Immunother. 2017;40(2):62-70.

28. Amara Suneetha, Venkataswarup Tiriveedhi. The five immune forces impacting DNA-based cancer immunotherapeutic strategy. Int $\mathrm{J} \mathrm{Mol}$ Sci. 2017;18(3). 